\title{
DESENVOLVIMENTO DE UM CUBESAT PARA DETECÇÃO DE DESCARGAS ATMOSFÉRICAS: PROJETO RAIOSAT
}

\author{
Antonio Cassiano Julio Filho ${ }^{1}$; Auro Tikami²; Elaine de Souza F. de Paula ${ }^{3}$; \\ Jhonathan Murcia Piñeros ${ }^{4}$; George Favale Fernandes ${ }^{5}$; Lázaro Pires Camargo ${ }^{6}$; \\ Carlos Alberto M. B. dos Santos ${ }^{7}$; Walter Abrahão dos Santos $^{8}$; Kleber Naccarato ${ }^{9}$
}

\author{
Instituto Nacional de Pesquisas Espaciais (INPE), São José dos Campos, São Paulo - Brasil \\ ${ }^{1}$ cassiano.filho, ${ }^{2}$ auro.tikami, ${ }^{3}$ elaine.paula, ${ }^{4}$ jhonathan.pineros, ${ }^{5}$ georgeff.sp, ${ }^{6}$ lazaro.camargo, \\ ${ }^{7}$ carlos.ambsantos, ${ }^{8}$ walter.abrahao, ${ }^{9}$ kleber.naccarato [@inpe.br]
}

\begin{abstract}
Resumo: Eventos atmosféricos severos são responsáveis por centenas de mortes e bilhões de dólares de danos anualmente em todo o mundo. No Brasil, diferentemente dos outros eventos hidrometeorológicos, os eventos atmosféricos severos são aleatórios e, assim, não apresentam padrão sócio espacial. Nesse contexto, há uma motivação significativa no sentido de aprimorar as técnicas de previsão desse tipo de evento utilizando modelos numéricos de alta resolução. Para isso, é necessária uma grande quantidade de dados observacionais de qualidade, entre eles dados de descargas atmosféricas em tempo quase real. Além disso, a detecção das descargas atmosféricas produzidas pelas tempestades é importante para uma ampla variedade de aplicações e em diversas áreas da pesquisa científica, que incluem o entendimento da ação do homem sobre o clima e como as mudanças climáticas podem afetar o comportamento das tempestades em longo prazo. Um dos métodos é a obtenção de dados através de satélites, neste sentido o objetivo do projeto RaioSat é desenvolver tecnologia nacional para detecção de descargas atmosféricas a partir do espaço a fim de complementar os dados existentes da rede de detecção de solo BrasilDAT. Este documento apresenta a metodologia para o desenvolvimento do sistema RaioSat.
\end{abstract}

Palavras-chave:Eventos Atmosféricos Severos, CubeSat, RaioSat.

\section{INTRODUÇÃO}

A detecção e geolocalização de descargas atmosféricas são importantes para uma ampla variedade de aplicações e em diversas áreas da pesquisa científica. Entre as principais, podemos citar os sistemas de previsão de curtíssimo prazo de ocorrência de eventos atmosféricos severos, identificação de danos em estruturas e definição de normas de proteção, análises de risco de acidentes envolvendo mortes e incêndios, pesquisa científica sobre fenômenos atmosféricos do sistema terrestre, determinação de impactos econômicos e sociais através de sinistros de seguro, interrupções de energia elétrica e dados forenses.

Os usuários típicos das informações de descargas atmosféricas incluem universidade, institutos de pesquisa, agências e institutos de meteorologia, empresas de aviação civil, órgãos militares (controle do tráfego aéreo e instalações de lançamento espacial), órgãos e agências ambientais e de gestão de recursos naturais, operadoras de transmissão e distribuição de energia elétrica.

Um sistema de detecção de solo é tipicamente constituído por uma rede composta por, no mínimo, quatro a cinco sensores e uma central de processamento. As redes de detecção de solo estão em operação desde a década de 1920, sendo que no Brasil uma rede de escala quase-continental, composta por mais de 60 sensores, denominada BrasilDAT, foi implementada a partir de 2010 e começou a produzir dados confiáveis para pesquisa científica e aplicações a partir de 2012 na região centro-sul do país. As regiões norte e nordeste (incluindo toda a Amazônia) continuam ainda descobertas.

As redes de detecção de descargas atmosféricas de solo possibilitam gerar dados precisos com altos custos de manutenção para garantir que todos os sensores, ou pelo menos a maioria, estejam operando, o que 
Antonio Cassiano Julio Filho; Auro Tikami; Elaine de Souza F. de Paula; Jhonathan Murcia Piñeros; George Favale Fernandes; Lázaro Pires Camargo; Carlos Alberto M. B. dos Santos; Walter Abrahão dos Santos; Kleber Naccarato

CubeSat para Detecção de Descargas Atmosféricas: Projeto RaioSat

exige boa infraestrutura de fornecimento de energia, disponibilidade de canais de comunicação e diversos requisitos de instalação em cada site. Os sensores a bordo de satélites permitem coberturas mais amplas, detectando descargas atmosféricas com a mesma eficiência e resolução temporais e espaciais.

Diante disso, há uma forte motivação para se aprimorar as técnicas de previsão de eventos atmosféricos severos usando modelos numéricos de previsão do tempo (do inglês, Numerical Weather Prediction models). Vários estudos publicados na literatura, sendo o principal realizado por (Fierro et al., 2012), mostram que a assimilação dos dados de descargas atmosféricas nos modelos de alta resolução espacial e temporal pode fornecer uma representação aprimorada da convecção no início do período de previsão.

Eventos atmosféricos severos (sistemas frontais transientes, complexos convectivos de mesoescala, ciclones tropicais, sistemas convectivos locais e tempestades tipo supercélulas) são responsáveis por dezenas de milhares de mortes e dezenas de bilhões de dólares de prejuízos anualmente em todo o mundo conforme dados publicados na internet pelo portal Our World in Data (https://ourworldindata.org/natural-disasters).

No Brasil, as condições atmosféricas que propiciam suas formações requerem maior aprofundamento para que seja possível a previsão de sua ocorrência com mais assertividade e tempo de antecedência (até 6 horas) para que alertas sejam emitidos aos diferentes segmentos da sociedade visando reduzir mortes e prejuízos materiais.

Atualmente o Instituto Nacional de pesquisas Espaciais (INPE), dentro de suas competências, pode promover meios de desenvolvimento tecnológico e científico atendendo demandas internas e externas através da coordenação de projetos, análise de missões, gerenciamento e incluindo a execução do projeto RaioSat, com sinergia entre instituições de ensino e pesquisas nacionais e internacionais, ou seja, "produzir ciência e tecnologia nas áreas espacial e do ambiente terrestre e oferecer produtos e serviços singulares em benefício do Brasil".

Este documento é organizado da seguinte forma: a seção 2 apresenta a Missão RaioSat, explanando sobre seu objetivo e detalha o segmento espacial e seus módulos; o segmento solo e o segmento lançador e suas considerações. A seção 3 apresenta os resultados esperados, a continuidade do projeto e trata de uma análise preliminar de uma constelação e finalmente a seção 4 apresenta as conclusões.

\section{A MISSÃO RAIOSAT}

A missão RaioSat pretende detectar simultaneamente descargas atmosféricas que ocorrem no céu (sem atingirem o solo), denominadas genericamente de intranuvem (Rakov \& Uman, 2003) e descarga que efetivamente atingem o solo, denominadas genericamente de nuvem-solo. A combinação dessas duas informações é chamada de dados de descargas atmosféricas totais.

Uma descarga atmosférica é essencialmente uma corrente elétrica impulsiva de grandes proporções que ocorre na troposfera da Terra como consequência da eletrificação (separação de cargas elétricas) das chamadas nuvens de tempestade ou cúmulos-nimbos e irradiam energia eletromagnética em uma ampla faixa de frequências (Naccarato, 2005).

A missão RaioSat pretende integrar pela primeira vez um sensor para detecção de raios em um CubeSat, com o desenvolvimento de tecnologia nacional para detecção de descargas atmosféricas a partir do espaço e complementar os dados existentes da rede de detecção de solo BrasilDAT, a qual não tem cobertura nacional e requer altos investimentos para se garantir um desempenho satisfatório. A detecção de descargas a partir do espaço (Nag et al. (2015), por sua vez, requer apenas um instrumento com ampla área de cobertura, o qual pode contribuir para ampliar a qualidade dos dados em todo território nacional com custos menores.

A Figura 1 apresenta uma visão geral com elementos da missão espacial RaioSat: Segmento Espacial, Segmento Solo e o Segmento Lançador. 
Figura 1. Elementos da Missão espacial RaiosSat, propriedades dos autores (2019)

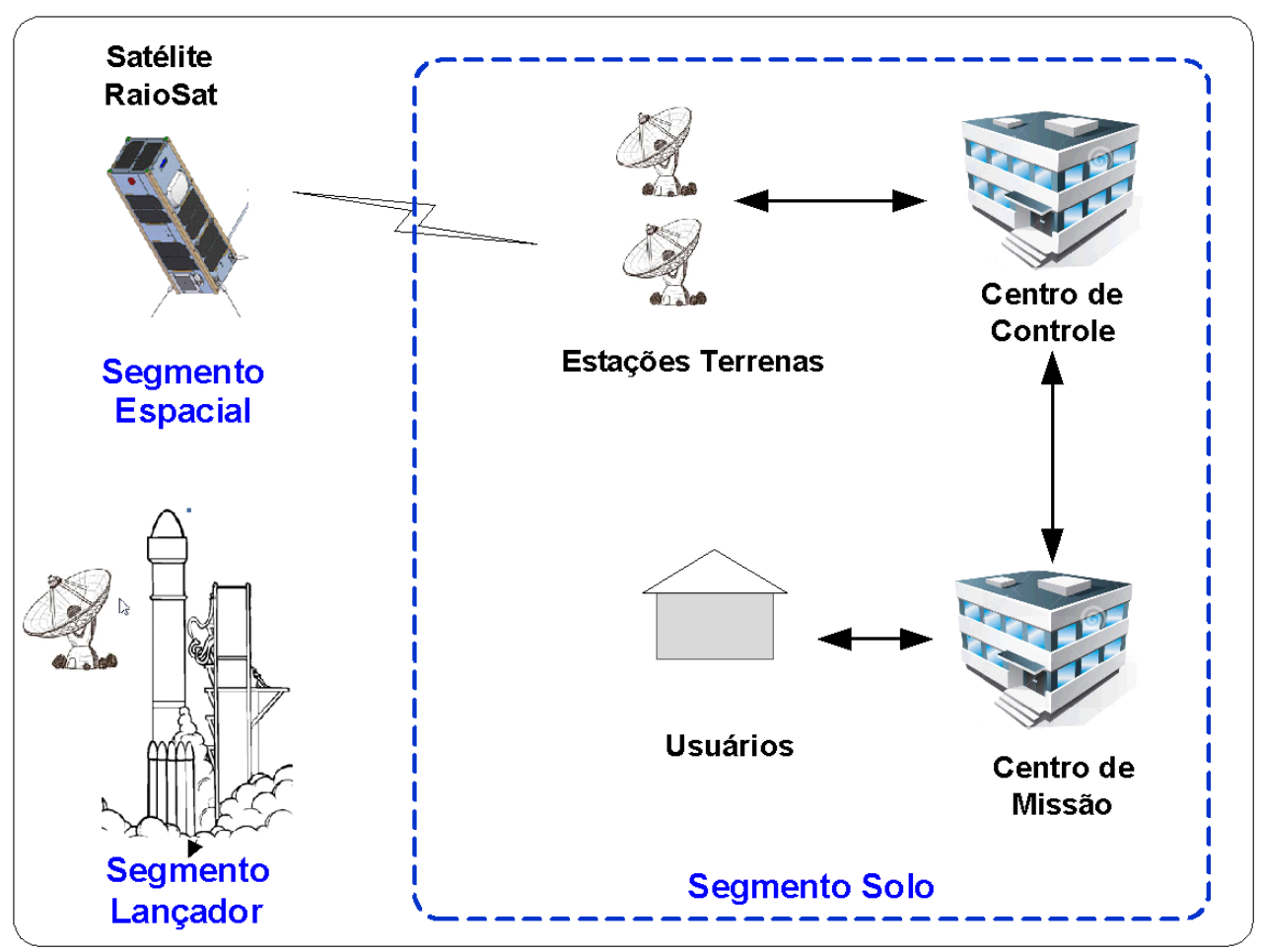

Os requisitos globais da missão como: dimensões, massa, consumo de energia, protocolos de comunicação, armazenamento de dados a bordo, controle de atitude, precisão de apontamento, órbita desejada e duração da missão são apresentados na Tabela 1.

Tabela 1. Requisitos globais da missão RaioSat

\begin{tabular}{|l|l|}
\hline \multicolumn{1}{|c|}{ Item } & \multicolumn{1}{c|}{ Requisito } \\
\hline Dimensões externas & CubeSat 3U $(10$ x 10 x $30 \mathrm{~cm})$ \\
\hline Massa total & até $3 \mathrm{~kg}$ \\
\hline Potência total consumida & $6,5 \mathrm{~W}$ (TBC) \\
\hline Telemetria UHF & Downlink até $9600 \mathrm{bps}$ \\
\hline Telecomando VHF & Uplink até $1200 \mathrm{bps}$ \\
\hline Protocolos de comunicação & $\begin{array}{l}\text { AX.25 (Radio amador), AFSK, FSK e } \\
\text { BPSK }\end{array}$ \\
\hline Quantidade de armazenamento total de dados a bordo & 4 Giga (TBC) com redundância \\
\hline Controle de Atitude & 3 eixos \\
\hline Precisão de apontamento & De 1 a 5 graus (TBC) \\
\hline Campo de visada da câmera (@ nível de nuvens) & 10 km \\
\hline Resolução espacial & 80 metros/pixel \\
\hline Resolução temporal & 500 quadros/segundo \\
\hline Órbita desejada & LEO \\
\hline Atitude & 650 Km \\
\hline Inclinação & $70^{\circ}(\mathrm{TBC})$ \\
\hline Duração da missão & $\begin{array}{l}\text { Mínimo de } 6 \text { meses, principalmente entre } \\
\text { outubro e março - temporada de tempestades }\end{array}$ \\
\hline Comprimento de onda desejável & 777,4 nm \\
\hline Sensibilidade da câmera & $400-750$ nm transmissão espectral \\
\hline
\end{tabular}


Antonio Cassiano Julio Filho; Auro Tikami; Elaine de Souza F. de Paula; Jhonathan Murcia Piñeros; George Favale Fernandes; Lázaro Pires Camargo; Carlos Alberto M. B. dos Santos; Walter Abrahão dos Santos; Kleber Naccarato

CubeSat para Detecção de Descargas Atmosféricas: Projeto RaioSat

Basicamente a missão operacional do RaioSat, inclui os seguintes passos:

a. O satélite monitora a ocorrência de tempestade de raios e coleta dados, como parte da missão global de detecção de raios.

b. As informações coletadas do raio são transmitidas para o sistema de tratamento de imagem.

c. Os dados são preprocessados a bordo para caracterizar a ocorrência de raio e também transmitidos para análise no centro de controle da missão.

d. O algoritmo de detecção de raios determina a probabilidade de sua ocorrência na localidade analisada.

e. Se os raios são detectados e validados, o sistema gera um conjunto de dados para os pesquisadores que indicam sua presença em local e horário específico.

f. As organizações usuárias reportam estatísticas de raios e notificam as autoridades responsáveis e/ou pesquisadores interessados. Os pesquisadores utilizam os dados em aplicações e fornecem seus resultados a outros usuários finais.

g. O sistema continua a monitorar novas ocorrências de raios em conjunto com a rede BrasilDat e a região na sua vizinhança.

Os modos de operação previstos para o RaioSat, incluindo o controle de órbita, requisitos de atitude e apontamento, ciclos ON/OFF são mostrados no diagrama de transição da Figura 2.

Figura 2. Modos de Operação do RaisoSat, propriedades dos autores (2019)

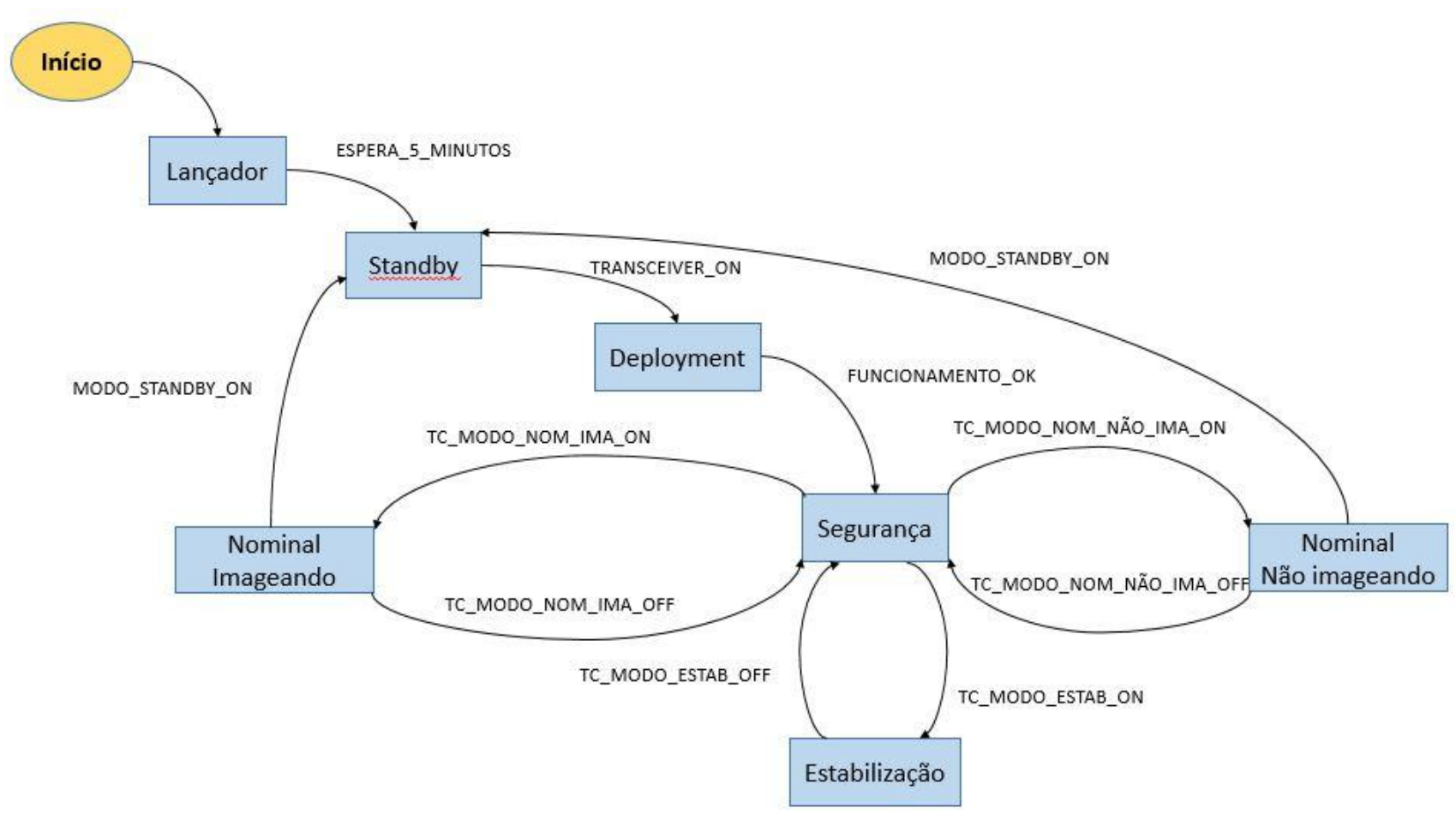




\subsection{Segmento Espacial}

O satélite RaioSat é baseado em uma estrutura CubeSat $3 \mathrm{U}(10 \times 10 \times 30 \mathrm{~cm})$, ilustrado na Figura 3, e será composto por uma plataforma principal, chamada de módulo de serviço (compreendendo os subsistemas de comunicações, suprimento de energia, computador de bordo e um controlador de atitude de 3 eixos) e um módulo de carga útil (Naccarato et al., 2017) O módulo de carga útil deverá contar com um receptor VHF e respectiva antena passiva na faixa de 80 a $200 \mathrm{MHz}$; uma câmera de imageamento espectral com resolução de 2.048 x 1536 pixels (Moura, 2017), e um receptor GPS.

Os estudos preliminares indicam uma órbita Low Earth Orbit (LEO) quase polar e duração da missão de no mínimo 6 meses (entre Outubro e Março) ou uma órbita LEO (com outra inclinação) de acordo com a disponibilidade e custos de lançamentos.

Figura 3. RaioSat com módulos de serviço e carga útil, propriedades dos autores (2019)

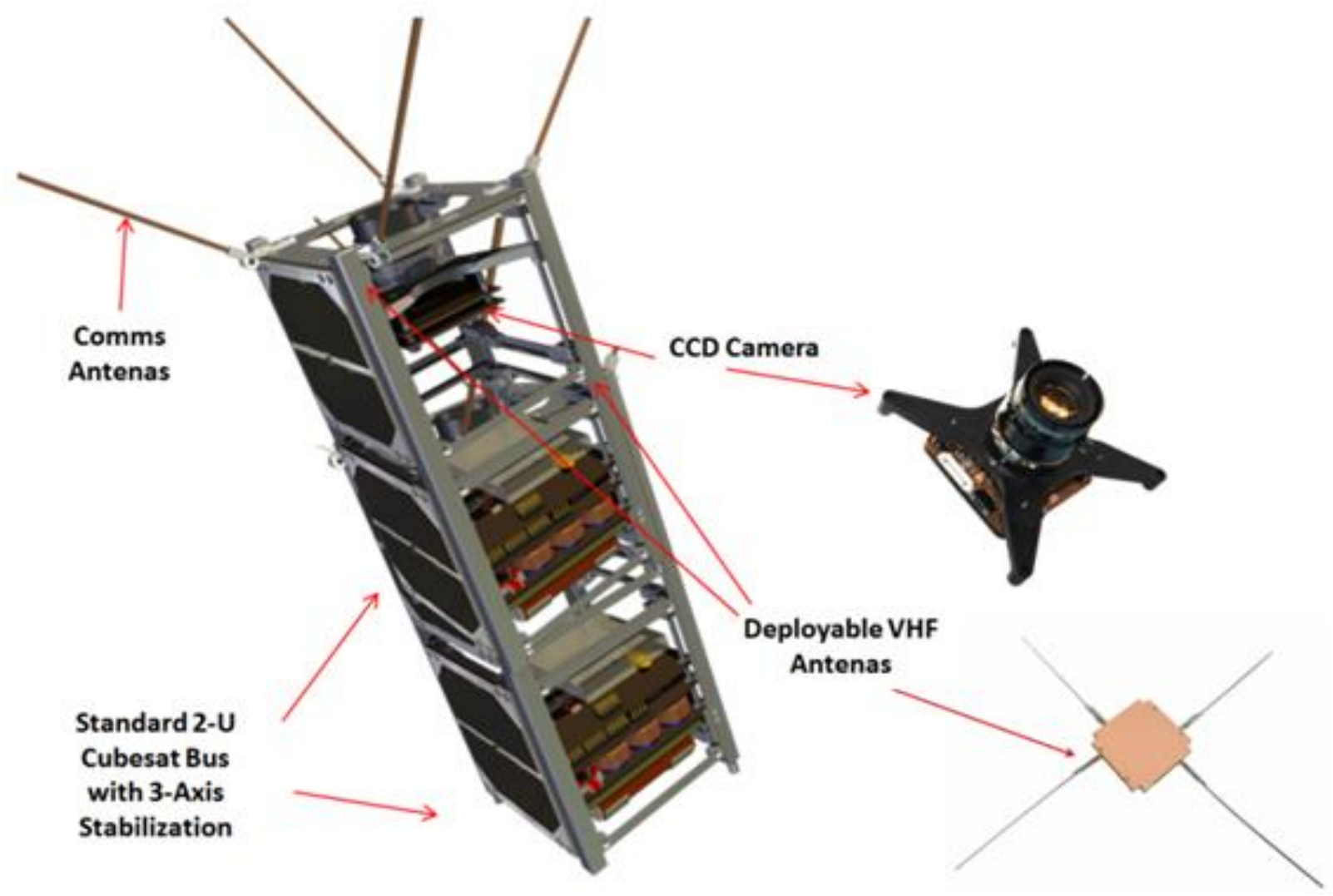

\subsubsection{Módulo de Carga Útil}

A câmera a ser utilizada deverá permitir uma imagem de superfície de $80 \mathrm{~m}$ por pixel, na faixa espectral de 700 a $900 \mathrm{~nm}$ usando um sensor com filtro ótico de passagem para as bandas OI (777, $4 \mathrm{~nm})$ e NII $(868,7$ nm) (Luz, 2015), e deverá atender às seguintes características: Resolução: 2048 x 1536 pixels; Sensor de imagem: RGB (Bayer) de 10 bits; RAM DDR2: 512 MB; Armazenamento: 2 GB; Formatos de saída: BMP, RAW e JPEG;Interfaces: CSP-enabled CAN, I2C, RS-422 e TTL.

O módulo de carga útil também deverá apresentar um receptor de VHF (Hamlin et al.,2009), na faixa de 80 a $200 \mathrm{MHz}$, para validar os eventos observados pela câmera através de um detector a ser implementado utilizando tecnologia de rádio definido por software (SDR). A Figura 4 ilustra um receptor VHF. 


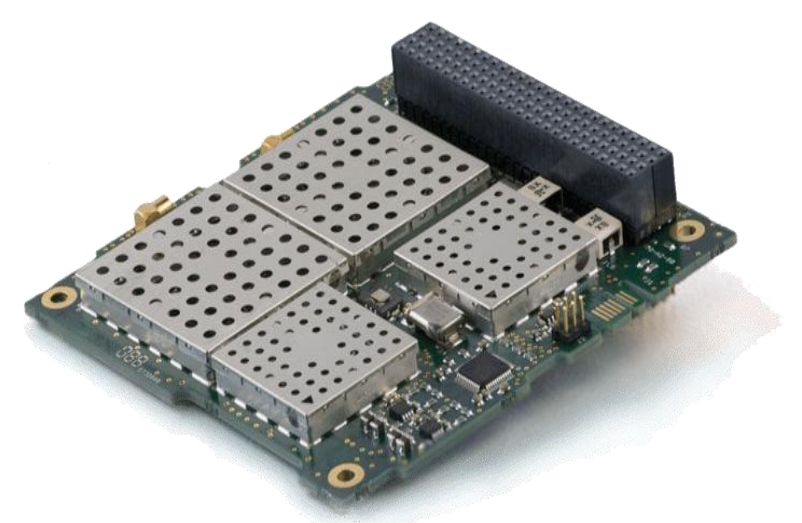

Para informação de tempo e georrefenciamento dos eventos, é necessário um receptor GPS a bordo do RaioSat. Uma opção de receptor GPS é o modelo piNAV-L1 da empresa Nano Avionics (www.navionics.com). O módulo GPSdeverá atender às seguintes características: Operação LEO (3600 km) , Consumo de $120 \mathrm{~mW}$ (típico), 3.3V @25드. GPS: sinal L1 C/A, 15 canais e taxa de posição de $1 \mathrm{~Hz}$.

\subsubsection{Módulo de Serviço}

a) Computador de Bordo

A computação de bordo do RaioSat será realizada pelo processador RISC da família ARM9 de 32bits e 400 MHz e é responsável pela realização das seguintes tarefas:

- Supervisão: Garantir que, em caso de falha, as ações de contingência sejam tomadas.

- Agendamento: Armazenar e realizar tarefas previamente agendadas por telecomandos.

- Recepção: Realiazar a demodulação e recuperação de dados de telecomandos.

- Housekeeping: Coletar as informações de diagnóstico dos subsistemas do satélite.

- Log: Gerar um registro dos eventos.

- Deployment: Distender as partes móveis do sistema.

- Beacon: Habilitar transmissão de sinal de beacon do RaioSat.

- Downlink: Transmitir a telemetria.

- Carga Útil: Gerenciar a atividade da carga útil.

Na Figura 5 é apresentada uma relação de tarefas do Computador de Bordo, do inglês On Board Computer, (OBC) de acordo com os modos de operação do RaioSat. 
Figura 5. Tarefas do Computador de Bordo (OBC)

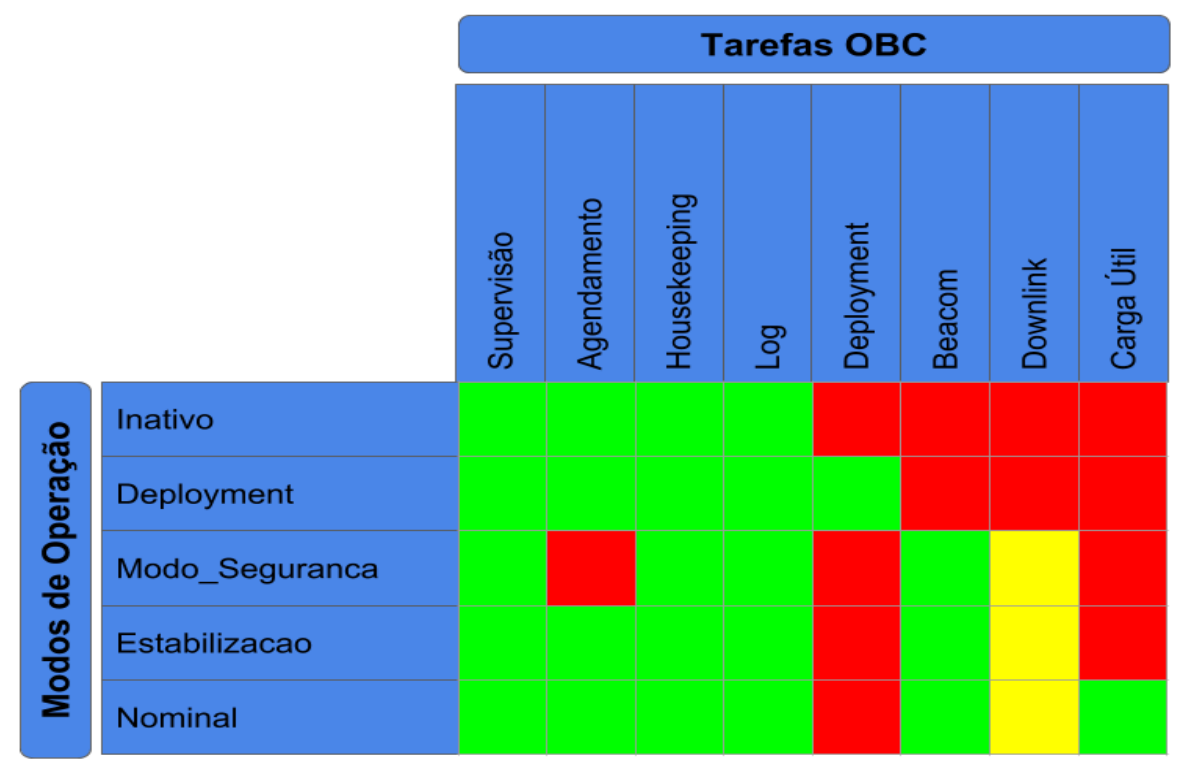

b) Suprimento de Energia

O subsistema de suprimento de energia, do inglês Electrical Power Subsystem, (EPS), segundo Mahdi et al, 2014, é responsável por gerar, armazenar, condicionar e distribuir a energia necessária para o funcionamento de todos os subsistemas e da carga útil durante a sua missão. O EPS pode ser dividido em 4 partes funcionais como ilustrado na Figura 6.

Figura 6. Arquitetura funcional do Subsistema de Suprimento de Energia Fonte: traduzido e adaptado de Wertz e Larson (1999)

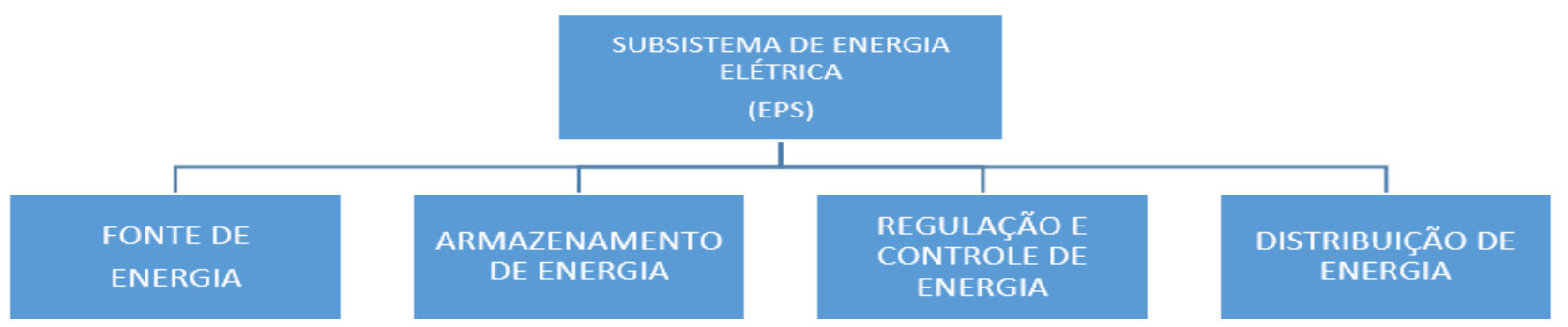

A primeira função, fonte de energia, está relacionada ao componente gerador de energia (painel solar) que em CubeSats, o mais comum faz uso de células solares fotovoltaicas.

A segunda função, armazenamento de energia, consiste em como será feito o armazenamento da energia sobressalente para suprir picos de consumo e o consumo durante os períodos de eclipses. Este armazenamento é feito por um conjunto de baterias.

A terceira função, regulação e controle de energia, determina como regulamos a potência de um satélite. Está relacionada com a arquitetura/método de transferência de energia, onde (Sullivan, 1989), cita as duas principais técnicas: rastreador de potência de pico (Power Peak Tracker ou PPT) e transferência direta de energia (Direct Energy Transfer ou DET) e detalha as topologias de EPS.

A Quarta função, distribuição de energia, está relacionado a forma como a energia será entregue às cargas. Consiste em conversores CA e reguladores DC. 
Antonio Cassiano Julio Filho; Auro Tikami; Elaine de Souza F. de Paula; Jhonathan Murcia Piñeros; George Favale Fernandes; Lázaro Pires Camargo; Carlos Alberto M. B. dos Santos; Walter Abrahão dos Santos; Kleber Naccarato

CubeSat para Detecção de Descargas Atmosféricas: Projeto RaioSat

O processo de projeto do EPS deve identificar as necessidades de início e fim de vida vinculando aos requisitos da missão. Os principais requisitos de alto nível para o RaioSat, adaptados de (Wertz \& Larson, 1999), são:

1. Fornecer uma fonte contínua de energia para as cargas durante a vida da missão.

2. Controlar e distribuir energia.

3. Garantir o atendimento das necessidades de consumo para situações comuns e de picos de consumo.

4. Prover conversores para CA e reguladores DC (se necessário)

5. Prover telemetrias para verificar a saúde e status do subsistema.

O balanço de potência varia de acordo com a missão. Segundo (Wertz \& Larson, 1999), as demandas de energia em fim de vida (EOL) devem ser reduzidas, a fim de se compensar a degradação do painel solar.

A Tabela2 mostra uma estimativa de demanda de potência do RaioSat para o processo de dimensionamento do painel solar e os demais componentes do EPS.

Tabela 2. Estimativa de potência consumida para o RaioSat

\begin{tabular}{|c|c|c|c|c|}
\hline \multirow{4}{*}{ Subsistema } & \multirow{4}{*}{ Equipamento } & \multicolumn{3}{|c|}{ Modos de Operação } \\
\hline & & \multirow{2}{*}{ Standby } & \multirow{2}{*}{$\begin{array}{l}\text { Nominal Não } \\
\text { Imageando }\end{array}$} & Nominal \\
\hline & & & & Imageando \\
\hline & & Potência $(\mathrm{mW})$ & Potência $(\mathrm{mW})$ & Potência $(\mathrm{mW})$ \\
\hline \multirow{3}{*}{ EPS } & Painel Solar & 0 & 0 & 0 \\
\hline & PCDU & 125 & 125 & 125 \\
\hline & Baterias & 0 & 0 & 0 \\
\hline OBC & CPU & 250 & 250 & 250 \\
\hline TT\&C & Antena UHF & 100 & 1000 & 1000 \\
\hline \multirow{2}{*}{ AOCS } & Magnetotorque & 0 & 1300 & 1300 \\
\hline & Rodas de Reação & 0 & 2700 & 2700 \\
\hline \multirow{4}{*}{ PAYLOAD } & $\begin{array}{l}\text { Câmera NanoCam } \\
\text { C1U }\end{array}$ & 0 & 0 & 330 \\
\hline & $\begin{array}{l}\text { Sensor PhotoBit } \\
\text { PB MV13 }\end{array}$ & 0 & 500 & 500 \\
\hline & GPS piNAV-L1 & 0 & 120 & 120 \\
\hline & Receptot VHF & 0 & 100 & 100 \\
\hline \multicolumn{2}{|c|}{ Subtotal $(\mathrm{mW})$} & 475 & 6095 & 6425 \\
\hline \multicolumn{2}{|c|}{ Total + margem $(20 \%)$} & \multicolumn{3}{|c|}{$7710 \mathrm{~mW}$} \\
\hline
\end{tabular}

De acordo com a Tabela 2, acima, no modo de operação Nominal Imageando, com todos os subsistemas ligados, a demanda de potência é de aproximadamente $6,5 \mathrm{~W}$. Os painéis solares deverão fornecer essa potência com uma margem de $20 \%$, ou seja, aproximadamente $7,7 \mathrm{~W}$.

c) Comunicações

O subsistema de comunicações, responsável pela troca de informações entre o satélite e o segmento solo, contém dois enlaces (uplink e downlink) que irão operar em banda UHF. A frequência de operação do RaioSat será coordenada pela União Internacional de Telecomunicações (ITU), órgão responsável pela coordenação global de radiofrequência.

Em satélites pequenos é bastante vantajosa que a comunicação entre satélite e o segmento solo ocorra dentro da faixa UHF de radioamador por satélite $(430$ a $440 \mathrm{MHz})$, pois os dados do RaioSat poderão também ser recebidos pela comunidade radioamadora. A União Internacional de Radioamadores (IARU) é responsável pela pré-coordenação de frequência para um satélite operando nessa faixa UHF citada. 
No telecomando (uplink) e telemetria (downlink) as taxas de dados serão, a priori, respectivamente 1200 e 9600 bps utilizando protocolo AX.25 e modulação GFSK.

A comunicação deverá ser realizada via transceptor half duplex programável na faixa de 430 a $440 \mathrm{MHz}$ de alto desempenho e baixo consumo. O receptor deverá ter alta sensibilidade (em torno de -118 dBm@9600 bps) e o transmissor deverá permitir comunicação de dados em canais de faixa estreita com modulação a ser definida entre GFSK, FM e FSK (incluindo G3RUH).

d) Sistema de Controle de Atitude

O controle de atitude em satélites é fundamental quando o apontamento se faz necessário como no uso de câmeras apontadas em direção à Terra. E também é fundamental no alinhamento de outros dispositivos como painéis solares e antenas em veículos espaciais em diferentes missões.

A precisão de apontamento da câmera do RaioSat será de até 5 graus com uma resolução espacial de 80 $\mathrm{m} /$ pixel e campo de visada de $10 \mathrm{~km}$ em órbita. O sistema de controle de atitude será realizado utilizando se principalmente bobinas magnéticas de torque em 3 eixos e rodas de reação também em 3 eixos.

\subsubsection{Estrutura Analítica do Produto e Arquitetura física}

A Estrutura Analítica do Produto (Product Breakdown Structure, PBS) do RaioSat é mostrada na Figura 7, analisando como os componentes dos subsistemas serão distribuídos.

A arquitetura física é adicionada à arquitetura física genérica, detalhando as características de desempenho e os requisitos de recursos para elementos físicos. Para a criação da arquitetura física, foi necessário alocar funções para a arquitetura genérica, atribuindo as interfaces entre os componentes físicos e derivados dos requisitos para aqueles componentes dos requisitos do sistema. Por exemplo, na arquitetura física instanciada, foram especificadas as rodas de reação (quantidade e capacidade), sensores (tipo e quantidade) e magnetômetros (quantidade e capacidade). A arquitetura física instanciada de RaioSat é mostrada na Figura 8.

Figura 7. Estrutura Analítica do Produto RaioSat, adaptado de Maia, et al., 2018

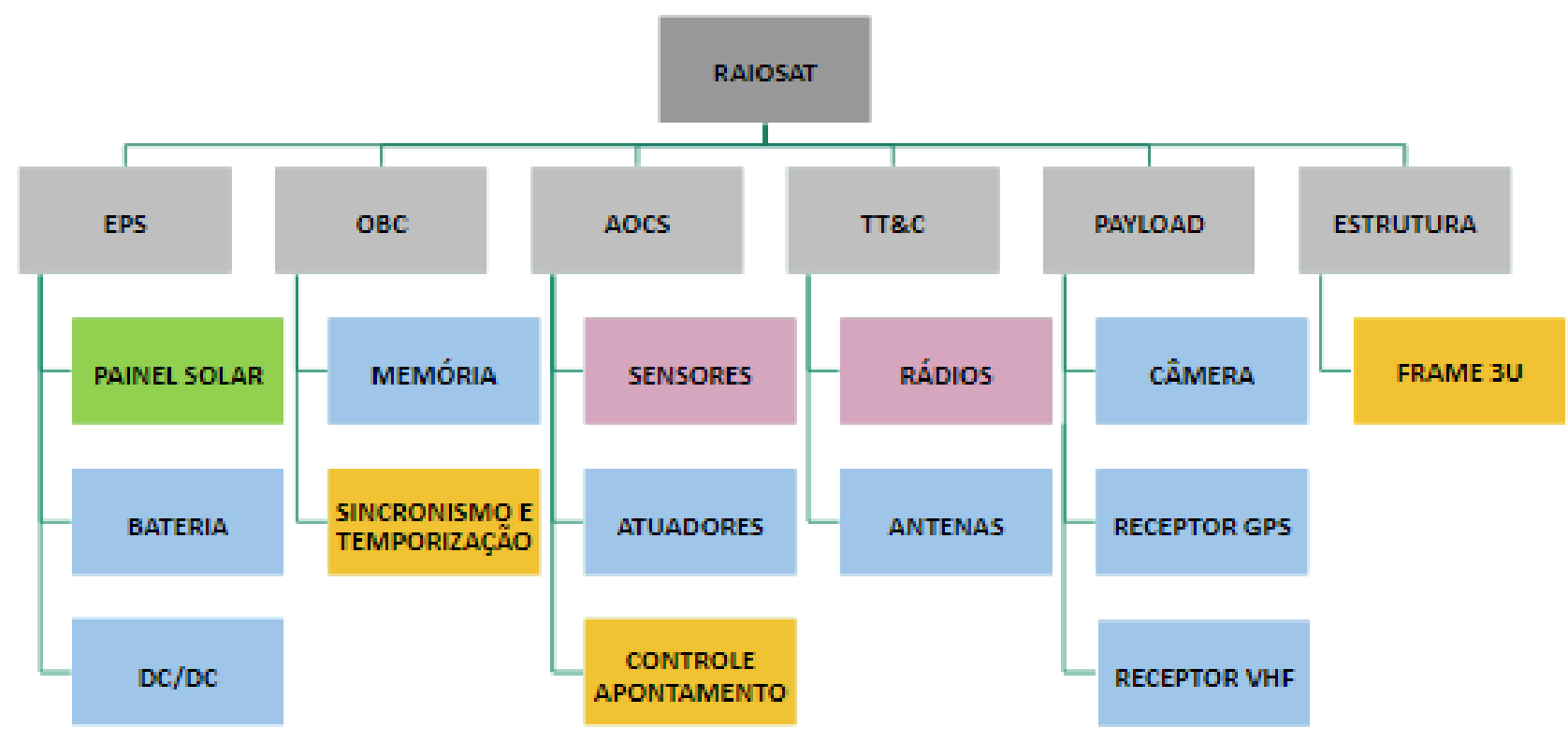


Antonio Cassiano Julio Filho; Auro Tikami; Elaine de Souza F. de Paula; Jhonathan Murcia Piñeros; George Favale Fernandes; Lázaro Pires Camargo; Carlos Alberto M. B. dos Santos; Walter Abrahão dos Santos; Kleber Naccarato

CubeSat para Detecção de Descargas Atmosféricas: Projeto RaioSat

Figura 8. Arquitetura física do RaioSat, , adaptado de Maia, et al., 2018)

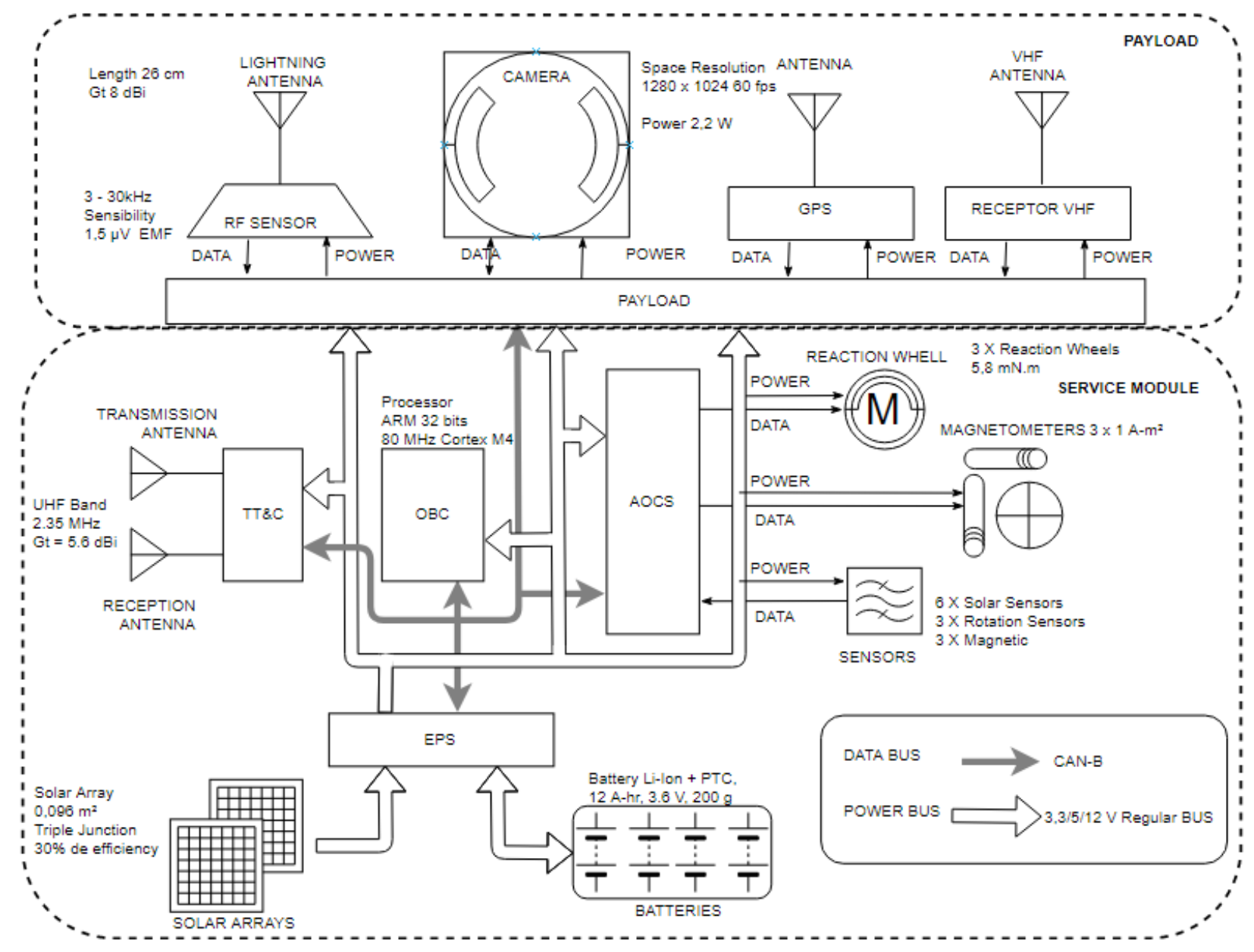

\subsection{Segmento Solo}

Para permitir a comunicação com o satélite, o INPE dispõe de duas estações terrenas para CubeSats localizadas em Santa Maria, RS e em São José dos Campos, SP, com as seguintes características: Antenas VHF/UHF Yagi, Amplificadores de baixo ruído e Terminal Node Controller (TNC) para 1200 - 9600 baud (AX.25 AFSK, FSK e BPSK); além das estações terrenas disponíveis, os dados também podem ser recebidos por radioamadores possibilitando uma ampla disseminação.

Os dados de telemetria de serviço e carga útil serão transferidos, via rede de dados, para o grupo de operação da missão e para o Grupo de Eletricidade Atmosférica (ELAT) do INPE. O RaioSat irá capturar informações de descargas atmosféricas que atingem o solo como a intensidade, a polaridade, o instante de ocorrência,e a localização geográfica. Essas informações serão enviadas para a Estação Terrena (ET) em operação, caso uma assinatura real de raio seja constatada. A ET receberá as detecções e processará estas informações distribuindo aos pesquisadores (usuários) através do portal do INPE.

\subsection{Segmento Lançador}

Para a definição do lançador a ser utilizado no programa RaioSat, serão necessárias várias análises sobre os veículos que poderão atender as especificações de altitude, inclinação, vida útil e os custos do lançamento. Entre as opções constam lançamentos a partir da Estação Espacial Internacional, do inglês International Space Station, (ISS) (limitada a sua órbita). Os CubeSats são levados à ISS em naves de transporte de suprimentos (como Cygnus, Dragon e Kounotori) lançadas por foguetes (como Falcon 9, Antares e H-IIB). 
Outros lançadores considerados são: o russo RS-20 (DNEPR), o americano Pegasus ou o Electron e o brasileiro VLM 1 .

Além deste conjunto de lançadores, existem várias empresas que organizam os lançamentos e, dessa forma, indicam as melhores opções de lançador para cada projeto de CubeSat. São exemplos dessas empresas a holandesa ISIS - Innovative Solutions In Space e a norte-americana Spaceflight Industries, responsável pelo programa The Smallsat Express.

A quantidade de lançadores para transportar numa inclinação de $70^{\circ}$ é reduzida em comparação com os lançadores para órbitas polares SSO $\left(98^{\circ}\right)$, onde existe o maior tráfego de CubeSats, principalmente para missões meteorológicas e de monitoramento.

Uma análise preliminar da órbita do RaioSat para cinco meses de missão, entre os meses de novembro de 2020 e abril de 2021, com máximas de luminosidade solar (12:00 - 17:00 UTC) e para um sensor com $45^{\circ}$ de FOV, mostra que o satélite tem 144 passagens pelo Brasil com tempo meio de 385s, passando pelo mesmo ponto a cada 6,5 dias.

Se a órbita do RaioSat for SSO, o número de passagens pelo território Brasileiro aumenta para 296 no mesmo período de tempo, com meia de duração de $403 \mathrm{~s}$ por passagem, onde o tempo médio de passagem pelo mesmo ponto é a cada 4.3 dias.

A escolha do lançador tem relação com os requisitos de qualificação espacial que incluem testes mandatórios e de desenvolvimento. Os testes mandatórios deverão ser realizados sob a imposição dos responsáveis pelo lançamento do CubeSat (Fernandes, 2014).

Os testes de desenvolvimento são realizados sob a demanda dos desenvolvedores a fim de garantir o funcionamento dos subsistemas quando expostos ao ambiente espacial (testes térmicos, de pressão, interferências eletromagnéticas), ou solicitações mecânicas durante seu transporte, integração e lançamento (choque mecânico, vibração, acústico) (Fernandes, 2016).

Neste sentido, será necessária a contratação de um laboratório, qualificado para testes espaciais. Neste caso o Laboratório de Integração e Teste (LIT), do INPE, mostra-se a melhor opção na relação custo $\mathrm{x}$ benefício, por sua localização, pela capacidade de realizar todos os testes mandatórios e por ser um laboratório qualificado para teste de sistemas espaciais.

\section{RESULTADOS ESPERADOS E CONTINUIDADE DO PROJETO}

Como principal resultado, tem-se a produção de dados confiáveis em várias áreas da pesquisa científica e no aprimoramento das metodologias de previsão de ocorrência de eventos severos, em sinergia com outras áreas de pesquisa e desenvolvimento da tecnologia nacional na área de detecção de descargas atmosféricas a partir do espaço com o aprimoramento de tecnologias existentes na área de pequenos satélites.

Os produtos gerados neste projeto multidisciplinar compreendem:

a) $\mathrm{O}$ avanço no conhecimento do efeito antropogênico sobre a formação e eletrificação das tempestades, o que inclui estudos de grandes centros urbanos e de queimadas de biomassa;

b) O desenvolvimento de métodos de previsão da ocorrência de eventos severos utilizando produtos de modelos numéricos (de previsão do tempo e eletrificação de nuvens) juntamente com dados observacionais (meteorológicos e elétricos) de superfície ou a bordo de satélite;

c) $\mathrm{O}$ entendimento de como as mudanças ambientais afetam (ou podem afetar) a formação e o regime das tempestades e, consequentemente, das descargas atmosféricas em mais longo prazo, particularmente nos ambientes urbanos.

Depois de validada a tecnologia para detecção de descargas atmosféricas através do RaioSat, o projeto deverá partir para a construção de outros satélites adicionais para que se obtenha uma constelação de, ao menos, 6 unidades. Essa constelação permitirá a cobertura de todo o território nacional, 24 horas por dia, 07 dias por semana, obtendo-se dados de descargas atmosféricas em quase tempo real com ampla cobertura espacial, boa resolução temporal e eficiência de detecção contínua e homogênea no espaço e no tempo. 
Antonio Cassiano Julio Filho; Auro Tikami; Elaine de Souza F. de Paula; Jhonathan Murcia Piñeros; George Favale Fernandes; Lázaro Pires Camargo; Carlos Alberto M. B. dos Santos; Walter Abrahão dos Santos; Kleber Naccarato

CubeSat para Detecção de Descargas Atmosféricas: Projeto RaioSat

\subsection{Análise Preliminar de uma Constelação para a missão RaioSat}

\subsubsection{Considerações}

As órbitas determinadas para a constelação da missão RaioSat são orbitas operacionais para fornecer monitoramento contínuo dos fenômenos meteorológicos. Os requisitos estabelecidos para o projeto da constelação é uma altitude de $650 \mathrm{~km}$, órbitas baixas com baixo custo para o lançamento. A órbita deve fornecer cobertura do território brasileiro e passagem para comunicação com as estações do INPE nas cidades de São José dos Campos e Santa Maria. Durante a vida em órbita os satélites não realizarão manobras de manutenção de altitude, mudança de plano e/ou decaimento porque não contarão com os subsistemas de propulsão necessários para realizar estas manobras.

Entre os desafios da missão, encontram se o aumento dos custos de lançamento, caso os satélites precisem de diferentes planos de órbitas, outro fator limitante é o custo total de desenvolvimento do projeto, isso limita a quantidade de satélites e número de órbitas, tentando ter a menor quantidade de lançamentos.

Pelos motivos mencionados, é analisada uma constelação de seis satélites. Como um dos objetivos da constelação é o aumento da cobertura da região de monitoramento, nos requisitos deve estar incluso as estações em solo para recepção de dados a cada passagem dos satélites. Se for otimizada, este tipo de constelação permite aumentar os tempos de contato com o satélite e a cobertura, porém, precisaria de diferentes planos de órbita o que incrementa os custos e tempos de lançamentos.

\subsubsection{Propostas Preliminares}

O estudo de uma constelação de seis satélites, distribuídos em três planos orbitais com dois satélites em cada plano, foi analisada por Carvalho et al, 2013. Este tipo de constelação apresenta o maior número de revisitas por dia e um bom número médio de contatos por dia, comparada com outras três constelações de 6 satélites com diferentes distribuições de órbita, entretanto requer três planos orbitais diferentes com dois satélites em cada um.

O aumento de lançamentos incrementa o custo da missão e o tempo entre lançamentos deve ser o menor, devido a que os satélites em LEO são altamente perturbados pelo arrasto atmosférico, gerando uma rápida variação dos elementos orbitais e mudando a cobertura e eficácia da constelação num curto período (em menos de um mês).

Uma constelação sol-síncrona (inclinação $97.98^{\circ}$ ) garante passagem constante pelo sol enquanto faz o monitoramento, permitindo um máximo de recarga de energia para o sensor e a transmissão de dados. A órbita sol-síncrona apresenta boas oportunidades de lançamento, mas, também conta com a maior quantidade de pequenos satélites, sendo uma órbita poluída por detritos espaciais (Anz-Meador, 2015). Este tipo de constelação é excelente para disponibilizar serviços para outros países, sendo o monitoramento global, reduzindo os custos e tempos de lançamento, requerendo a cooperação para maior distribuição de estações.

Para o caso do RaioSat, a constelação proposta é de seis satélites espaçados a cada $60^{\circ}$ num plano com inclinação de $30^{\circ}$, órbita circular e altitude de $650 \mathrm{~km}$. Num dia de órbita é possível ter mais de 55 passagens pelo Brasil, tendo $100 \%$ de cobertura do território durante o dia e a noite. O maior gap é de $8 \mathrm{~h}$, quando os satélites encontram se no ponto de máxima latitude pela inclinação, acontece uma vez por dia. A constelação permite uma cobertura de mais de 16 horas com gaps ao redor de 10 minutos. Este tipo de constelação garante $100 \%$ de cobertura do país diariamente, aumentando a capacidade de um único satélite, reduzindo os custos de lançamento porque está planejada para posicionar todos os satélites num plano orbital, permitindo a disponibilização de serviços para outros países localizados em latitudes entre $-30^{\circ}$ até $30^{\circ}$. Para reduzir os gaps e aumentar os tempos de contato é necessária pelo menos uma estação no norte do país para aumentar a capacidade de coleta de dados.

Na figura 9 é apresentado o tempo de cobertura de cada um dos satélites da constelação durante um dia de operações. A figura mostra o contato dos sensores dos satélites desde o número 1 até o número 6 em 
diferentes cores. O eixo horizontal representa o tempo desde a data da simulação em segundos (Epoch) até 24h depois, ou $86.400 \mathrm{~s}$. Na figura é possível observar uma continua cobertura do território brasileiro com alguns intervalos ao redor de 200 segundos entre satélite e satélite. A região branca da figura indica falta de cobertura dos sensores do satélite e acontece quando os satélites passam pela maior latitude da órbita no mesmo instante que o Brasil passa embaixo deles. A redução da inclinação da órbita permite aumentar a quantidade de contatos, porém reduz a cobertura do país ( menor do que 90\%). As passagens foram simuladas na versão de testes do software AGI/STK.

Figura 9. Simulação dos contatos entre os sensores dos satélites e o território brasileiro para um dia de órbita

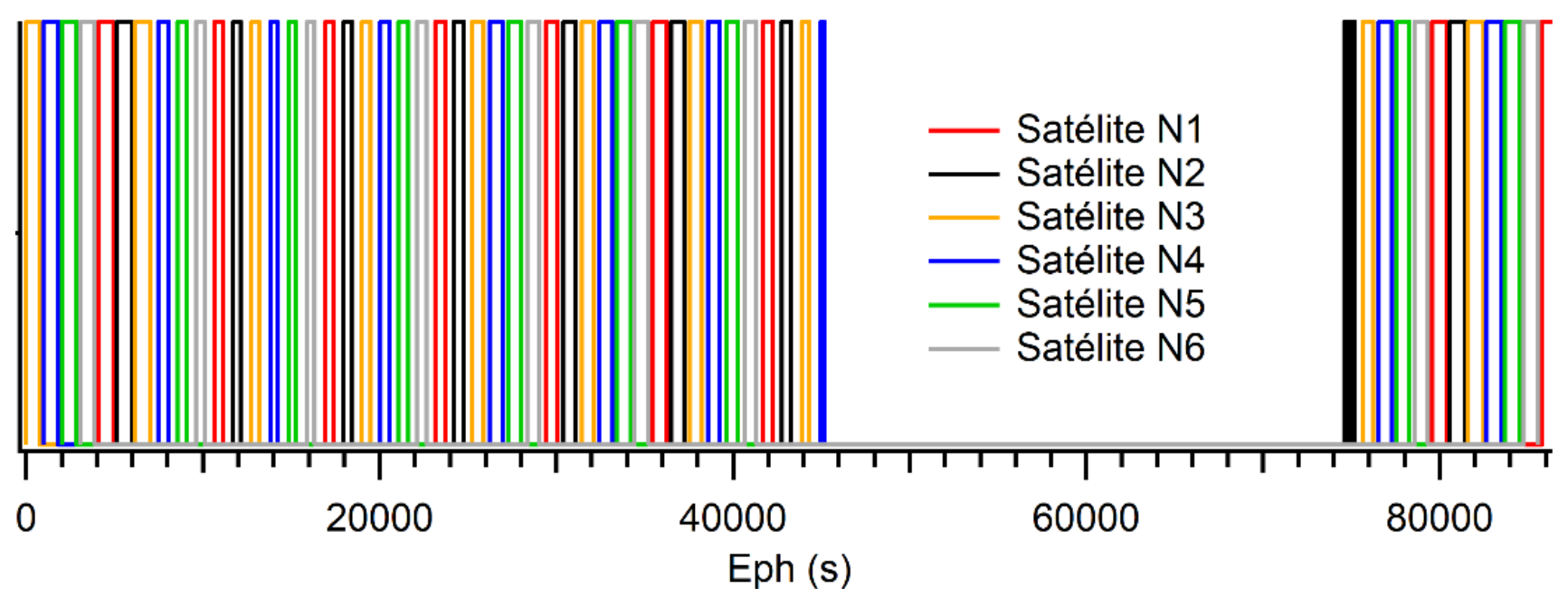

\section{CONCLUSÕES}

No Brasil, as condições atmosféricas que propiciam suas formações de descargas atmosféricas são pouco estudadas e requerem maior conhecimento para que seja possível a previsão de sua ocorrência com mais assertividade e tempo de antecedência para que os alertas sejam emitidos aos diferentes segmentos da sociedade visando reduzir mortes e prejuízos materiais. Dessa forma, a detecção de dados de descargas atmosféricas para todo território nacional de maneira uniforme será uma importante contribuição para o aprimoramento das técnicas de previsão de eventos severos de curtíssimo prazo (até 6 horas).

O principal resultado é a produção de dados confiáveis que serão úteis em várias áreas da pesquisa científica e no aprimoramento das metodologias de previsão de ocorrência de eventos severos, em sinergia com outras áreas do INPE.

Além da produção de dados confiáveis o projeto RaioSat pode promover meios de desenvolvimento tecnológico e científico com sinergia entre instituições de ensino e pesquisas nacionais e internacionais, ou seja, "produzir ciência e tecnologia nas áreas espacial e do ambiente terrestre e oferecer produtos e serviços singulares em benefício do Brasil".

O resultado técnico mais importante e inovador do trabalho é que a metodologia apresentada garante a integração, pela primeira vez, de um sensor para detecção de raios em um CubeSat.

O trabalho também apresenta uma análise preliminar de uma constelação para aumento da cobertura e permitir a disponibilização de serviços para outros países. 
Antonio Cassiano Julio Filho; Auro Tikami; Elaine de Souza F. de Paula; Jhonathan Murcia Piñeros; George Favale Fernandes; Lázaro Pires Camargo; Carlos Alberto M. B. dos Santos; Walter Abrahão dos Santos; Kleber Naccarato

CubeSat para Detecção de Descargas Atmosféricas: Projeto RaioSat

\section{REFERÊNCIAS}

Anz-Meador, P. A review of space environment implications of cubesat traffic 2003-2014. Orbital Debris Quarterly News, v. 19, n. 3, p. 4-6, 2015.

Carvalho, M., Lima, J., Jotha, L., Aquino, P. CONASAT- Constelação de Nano Satélites para Coleta de Dados Ambientais. Em; Anais XVI Simpósip Brasileiro de Sensoriamento Remoto - SBSR, Foz do Iguaçu, PR, Brasil 13 - 18 de abril de 2013. Pp. 9108 - 9115.

Fernandes, G. F., 2014."Procedimento de Testes Vácuo-térmicos, Picosatellite Tancredo I - PFM, Acceptance Level, Program UBATUBASAT, IT01-CUBSAT-TP-001", Private Communication, Issue 1.

Fernandes, G. F.; Santos, M. B. ; Almeida, J. S. ; Nogueira, P. R. M. ; Silva, V. D., 2016. "Thermal Tests for CubeSat in Brazil: lessons learned and the challenges for the future". In: 67th International Astronautical Congress - IAC, Guadalajara.

Fierro, A. O., Mansell, E. R., Ziegler, C. L., \& MacGorman, D. R., 2012. "Application of a lightning data assimilation technique in the WRF-ARW model at cloud-resolving scales for the tornado outbreak of 24 May 2011". Monthly Weather Review, 140(8), 2609-2627.

Hamlin, T.; Wiens, K.C.; Jacobson, A.R.; Light, T.E.L.; Eack, K.B. Space- and ground-based studies of lightning signatures. In: Lightning: principles, instruments and applications - Review of modern lightning research, Ed.: H. D. Betz, U. Schumann, P. Laroche, Springer, 641 pp., 2009.

Luz, E. S. A. M. Estudo de casos de descargas atmosféricas utilizando espectroscopia sem fenda (slitless). 2015. 198 p. (sid.inpe.br/mtc-m21b/2015/11.30.02.40-TDI). Dissertação (Mestrado em Geofísica Espacial/Ciências Atmosféricas) - Instituto Nacional de Pesquisas Espaciais (INPE), São José dos Campos, 2015. Disponível em: <http://urlib.net/rep/8JMKD3MGP3W34P/3KMAELH>.

Mahdi, M. C.; Jaafer, J. S.; Shehab, A.-A.-R. Design and Implementation of an Effective Electrical Power System for Nano-Satellite.International Journal of Scientific \& Engineering Research, [S.1.], v.Volume 5, n.Issue 5, p.29-, May 2014.

Maia, G. F. S.; Paula, E. S. F.; Pereira, M. O.; Camargo, L. A. P.; Naccarato, K. P.; Dos Santos, W. A., 2018. "High-level Developments in Space Systems Engineering of the RaioSat Project". In: 3nd Latin American Cubesat Workshop (LACW), Ubatuba, Brazil.

Moura, C. O. "Análise de viabilidade de uma carga útil óptica em nanossatélites para detecção de raios". 2017. 153 p. IBI: <8JMKD3MGP3W34P/3PA5A98>. (sid.inpe.br/mtc-m21b/2017/07.17.13.49-TDI). Dissertação (Mestrado em Engenharia e Gerenciamento de Sistemas Espaciais) - Instituto Nacional de Pesquisas Espaciais (INPE), São José dos Campos, 2017. Disponível em: <http://urlib.net/rep/8JMKD3MGP3W34P/3PA5A98>.

Naccarato, K. P., 2005. "Análise das características dos relâmpagos na região sudeste do Brasil”. Tese de Doutorado em Geofísica Espacial, Instituto Nacional de Pesquisas Espaciais (INPE), São José dos Campos, Brasil. Disponível em: <http://urlib.net/6qtX3pFwXQZGivnJSY/HB8iH>.

Naccarato, K. P. et al, 2017. "The RaioSat project: detecting total lightning flashes from a CubeSat", 2nd IAA Latin American CubeSat Workshop (LACW), Ubatuba.

Nag, A.; Murphy, M. J.; Schulz, W.; Cummins, K. L. (2015). Lightning locating systems: Insights on characteristics and validation techniques. Earth and Space Science, 2(4), 65-93. DOI: 10.1002/2014EA000051.

Rakov, V.A.; Uman, M.A. (2003) "Lightning: physics and effects". Cambridge University Press, 687 pp.

Sullivan, D. O., 1989. "Satellite power system topologies". ESA Journal, v.13, p.77-88, 1989b.

Wertz, J. R., Larson W. J., 1999. "Space Mission Analysis and Design". Microcosm, 976 pp; 3rd edition.p.407-427. 